\title{
BMJ Open HOspitals and patients WoRking in Unity (HOW R U?): telephone peer support to improve older patients' quality of life after emergency department discharge in Melbourne, Australia-a multicentre prospective feasibility study
}

To cite: Lowthian JA, Lennox A, Curtis A, et al. HOspitals and patients WoRking in Unity (HOW $R U$ ?): telephone peer support to improve older patients' quality of life after emergency department discharge in Melbourne, Australia-a multicentre prospective feasibility study. BMJ Open 2018;8:e020321. doi:10.1136/ bmjopen-2017-020321

- Prepublication history and additional material for this paper are available online. To view these files, please visit the journal online (http://dx.doi. org/10.1136/bmjopen-2017020321).

Received 31 0ctober 2017 Revised 4 May 2018 Accepted 14 May 2018
Check for updates

For numbered affiliations see end of article.

Correspondence to Dr Judy A Lowthian; Judy.lowthian@monash.edu

\section{ABSTRACT}

Objectives To ascertain the feasibility and acceptability of the HOW $R U$ ? programme, a novel volunteer-peer postdischarge support programme for older patients after discharge from the emergency department (ED).

Design A multicentre prospective mixed-methods feasibility study.

Setting Two tertiary hospital EDs in metropolitan Melbourne, Australia.

Participants A convenience sample of 39 discharged ED patients aged 70 years or over, with symptoms of social isolation, Ioneliness and/or depression.

Intervention The HOW $R$ U? intervention comprised weekly social support telephone calls delivered by volunteer peers for 3 months following ED discharge. Primary and secondary outcome measures The primary outcomes were feasibility of study processes, intervention acceptability to participants and retention in the programme. Secondary outcomes were changes in loneliness level (UCLA-3-3-item Loneliness Scale), mood (5-item Geriatric Depression Scale) and health-related quality of life (EQ-5D-5L and EQ-VAS) postintervention. Results Recruitment was feasible, with $30 \%$ of eligible patients successfully recruited. Seventeen volunteer peers provided telephone support to patient participants, in addition to their usual hospital volunteer role. HOW $R$ $U$ ? was well received, with $87 \%$ retention in the patient group, and no attrition in the volunteer group. The median age of patients was 84 years, $64 \%$ were female, and $82 \%$ lived alone. Sixty-eight per cent of patients experienced reductions in depressive symptoms, and $53 \%$ experiencing reduced feelings of loneliness, and these differences were statistically significant Patient feedback was positive and volunteers reported great satisfaction with their new role. Conclusion HOWR U? was feasible in terms of recruitment and retention and was acceptable to both patients and volunteers. The overall results support the potential for further research in this area and provide data
Strengths and limitations of this study

This is the first feasibility study of a hospital volunteer-delivered telephone service to support older people with symptoms of social isolation, loneliness and/or depression after discharge from the emergency department.

- Recruitment and retention rates support the feasibility of the intervention.

- Reductions in loneliness and depressive symptoms support further research to test the intervention in a definitive trial.

- This was a relatively small cohort study, hence a randomised controlled trial is required to confirm the observed effects.

to support the design of a definitive trial to confirm the observed effects.

Trial registration number ANZCTRN12615000715572; Results.

\section{INTRODUCTION}

Older people presenting to emergency departments (EDs) and hospitals have a higher likelihood of social isolation, loneliness and depression ${ }^{1-3}$; all of which are associated with negative health outcomes, functional decline, institutionalisation, mortality and increased hospital use. ${ }^{4-9}$

These risk factors for increased hospital use and poor health outcomes are not routinely screened for during ED attendances or short hospital admissions other than in the research setting. Despite this, ED attendances 
represent an opportunity to identify older patients who are at risk of further negative health outcomes and increased acute health service use. Targeted management of older people suffering from social isolation, loneliness or depressive symptoms has been shown to be effective in reducing symptoms. ${ }^{5}$ It is highly probable that systematic identification of isolation, loneliness and depressive symptoms at the time of ED attendance, with postdischarge support, will help combat these negative consequences and diminish this important public and individual health burden.

Peer support is the "provision of knowledge, experience, emotional or practical help by someone sharing common characteristics. ${ }^{10}$ Peer support can be used with patients transitioning from hospital to home to enhance quality of life. This definition falls within the social support model and postulates that social relationships promote health and well-being; thus peer support is hypothesised to reduce feelings of social isolation and loneliness, thereby improving well-being. ${ }^{11}$

Peer support is provided by a person sharing common characteristics (eg, age, gender, socioeconomic status, ethnicity, or experience of acute illness and hospitalisation). Equivalent 'status' between peer and patient is a feature of peer support that facilitates a high level of empathy delivered in a non-confrontational manner. ${ }^{12}$ Peers may be hospital volunteers who are trained to support and listen, but not to give medical advice or judgement. This non-medical status helps overcome any reluctance that patients may have in discussing feelings of loneliness or isolation, thus helping to bridge the gap between patients and health professionals. ${ }^{13}$ Peer support can be delivered via home visits, group meetings or telephone calls.

The aim of this study was to test the feasibility and acceptability of HOspitals and patients WoRking in Unity (HOW R U?), a postdischarge, telephone peer support intervention delivered by hospital volunteers to older community-dwelling patients with feelings of social isolation, loneliness or depression. If the intervention is feasible and acceptable, the findings will inform design and conduct of a randomised controlled trial and programme evaluation.

\section{METHODS}

\section{Patient and public involvement statement}

This study was informed by comments received from patient participants in the Safe Elderly Emergency Discharge (SEED) project. SEED mapped the demographic, clinical, functional and psychosocial profiles of a large cohort $(n=959)$ of older ED patients. The cohort was followed up by telephone over a 6-months period after discharge home to determine the risk factors associated with adverse outcomes. ${ }^{89}$ Many patients reported how much they looked forward to the follow-up calls with requests for more frequent calls, highlighting their feelings of isolation and loneliness. This led to the development of our hypothesis that telephone support could reduce feelings of social isolation, loneliness and depression. Potential patients and hospital-based volunteers were involved in the development of the HOW R U? intervention, with volunteers directly involved as research partners in all aspects of the study (GW, CR). Hospital-based volunteers were involved in conduct of this study, including development and publication of the study protocol and this manuscript. ${ }^{14}$ Patients from the current feasibility and acceptability study have been involved in refinement of study processes and of the intervention for the planned RCT.

\section{Design, setting and participants}

This was a pragmatic prospective mixed-methods feasibility study conducted with a cohort of patients following discharge home from the EDs of two tertiary hospitals. The Alfred and Cabrini Hospitals provide public and private healthcare in metropolitan Melbourne, respectively. Participants were community-dwelling patients aged 70 years or more, who attended The Alfred ED between November 2015 and March 2016, and Cabrini ED between March and July 2016, and were discharged home from the ED, short-stay observation unit or acute medical ward within 72 hours of arrival. Patients were recruited on weekdays throughout the study period by research nurses. All participants gave written informed consent.

Eligible patients had symptoms of social isolation, loneliness and/or depression using the Social Isolation Index $(\mathrm{SII} \geq 3),{ }^{15}$ 3-item Loneliness Scale $(\mathrm{UCLA}-3 \geq 6)^{16}$ and 5-item Geriatric Depression Scale (GDS-5 $\geq 2$ ). ${ }^{17}$

Patients were excluded if they were triaged as category 1 level of urgency on the Australasian Triage Scale, required surgery, lived in an aged care facility, were receiving end-of-life care, had a confirmed diagnosis of dementia or severe mental illness such as psychosis or schizophrenia, had a moderate to severe cognitive impairment using the Mini-Mental State Examination $(<20),{ }^{18}$ or were unable or unwilling to communicate by telephone.

\section{Sample size}

A sample size of 50 participants across the two sites was nominated to examine feasibility of study processes and intervention acceptability.

\section{HOW R U? intervention}

The intervention, volunteer peer training programme and risk management strategies were described in full in the published study protocol. ${ }^{14}$ In summary, HOW R U? comprised:

- Screening by research nurses for feelings of social isolation, loneliness and depression at the time of hospital attendance using the SII, ${ }^{15}$ UCLA- ${ }^{16}$ and GDS-5. ${ }^{17}$

- Peer support delivered by a trained hospital volunteer through weekly telephone calls, within 72 hours of discharge home, for up to 3 months. 
- Referral for ongoing support by community-based services as required at study end.

\section{Data collection}

As per the published study protocol paper, ${ }^{14}$ bio-sociodemographic and health and social care services use data were collected, alongside measurement of social isolation (SII),${ }^{15}$ loneliness (UCLA-3), ${ }^{16}$ depressive symptoms $(\text { GDS-5 })^{17}$ and health-related quality of life (EQ-5D-5L and EQ-VAS) ${ }^{19}$ at the time of hospital attendance and at the 3 months study endpoint. The primary outcomes were feasibility and acceptability.

Feasibility of study processes including recruitment and retention in the programme were assessed using study records. Thirty-nine patient experience interviews were conducted at the conclusion of follow-up data collection to determine the acceptability of the intervention. These interviews were undertaken using a topic guide based on the Peer Support Evaluation Inventory. ${ }^{20}$ Questions explored participants' perceptions about the frequency and length of the calls, the modality of the intervention, their matched volunteer peers, the level of support provided and their satisfaction with the overall experience (online supplementary appendix). Fidelity of the intervention delivery was determined by reviewing the weekly telephone activity logs maintained by the volunteer peers and also through observation of a proportion of peer support calls. Secondary outcomes were any measurable changes in levels of perceived social isolation, loneliness, depressive symptoms and quality of life.

\section{Analysis}

Acceptability of the intervention by the target patient population was measured by the rate of recruitment and retention in the intervention and also through analysis of the qualitative interviews. Transcripts were loaded into NVivo (V.11; QSR International, Doncaster, Victoria, Australia) for data management and analysed using a qualitative thematic framework approach. ${ }^{21}$ This involved familiarisation with the data and derivation of a framework by noticing concepts within the data and developing themes and subthemes. Quotes were sorted into categories, which formed the final thematic framework. Data were mapped and interpreted and the framework was applied back to the dataset to ensure all quotes were appropriately organised while retaining links to the original data. Two researchers were involved in the development of the framework and resolved differences in opinion through discussion.

Acceptability to volunteer peers was measured using retention rates and feedback obtained in focus groups. Volunteer perceptions are the focus of a separate manuscript.

Social isolation, loneliness, depressive symptoms and health-related quality of life scores were compared before and after the intervention, using paired t-tests with a significance level of $\mathrm{p}=0.05$.
Table 1 HOW R U? participant baseline demographic characteristics

\begin{tabular}{ll}
\hline & $\mathbf{n = 3 9}$ \\
\hline Age (years), median (range) & $84(70-100)$ \\
Sex & \\
$\quad$ Female & $64 \%$ \\
Cultural background & \\
$\quad$ Australian born & $77 \%$ \\
Living status & \\
Living alone & $82 \%$ \\
Formal/informal care in place & $44 \%$ \\
Regular social group attendance & $53 \%$ \\
Feelings of social isolation (SII $\geq 2)$ & $82 \%$ \\
Feelings of loneliness (UCLA-3 26$)$ & $65 \%$ \\
$\quad$ Depressive symptoms (GDS-5 $\geq 2)$ & $77 \%$ \\
Self-rated health: EQ-VAS (average) & 59.6 \\
\hline
\end{tabular}

GDS-5, five-item Geriatric Depression Scale; SII, Social Isolation Index; UCLA-3, 3-Item Loneliness Scale.

\section{RESULTS}

This study enabled us to develop all study resources, materials and training programmes; test the feasibility of study processes and determine acceptability of the intervention to patients and volunteers. We recruited 17 volunteer peers and a convenience sample of 39 patient participants. Volunteers were all aged over 50 years and $69 \%$ were women. The median age of patient participants was 84 years, $64 \%$ were women and $84 \%$ of participants lived alone. Patient participant baseline demographic characteristics are summarised in table 1.

\section{Feasibility of study processes}

Volunteers were invited by their hospital volunteer services manager to participate in the study. All volunteer participants attended a half-day HOW $R U$ ? peer support training programme, conducted at their respective hospital. Feedback about the first hospital's HOW R U? orientation/training programme and resources enabled refinement prior to the second hospital's session.

Recruitment processes in the ED, including eligibility screening, were feasible, with $30 \%$ of eligible patients successfully enlisted across the two sites.

\section{Intervention acceptability and fidelity}

The intervention was feasible and acceptable from the volunteers' point of view, with most able to take on three participants in addition to their usual hospital volunteer roles. There was no volunteer attrition over the study period. The mean number of telephone calls per participant was 7.73 calls (SD 2.71), with a mean call length of $23.97 \mathrm{~min}$ (SD 13.39). Weekly monitoring of telephone activity logs indicated intervention fidelity, with $100 \%$ completion rate of the activity log sheets including documentation of the main focus of and topics discussed in 
each call; agreed social goals for the following week; patient-reported changes since the previous call and volunteer-peer impression of the participant's emotional state/feelings during each call. All volunteers adhered to the risk management procedures in accordance with the study protocol, with one volunteer reporting concern about a single patient participant to the hospital emergency physician coinvestigator. All volunteers reported that the level of support provided by their volunteer service manager and the research team was appropriate.

The intervention was acceptable to patient participants, with 34 completing the programme, representing an $87 \%$ retention rate. Three main themes identified in the qualitative data were as follows:

\section{Study processes were acceptable to participants}

While some participants missed a few calls due to lastminute medical appointments and unexpected visitors, the fact that participants agreed on the call time the week prior meant that receiving peer-support calls was convenient for them. Participants were satisfied with the individually determined length of their phone calls, with one expressing that 'having someone to talk to for 5-10 min is good' (P13) while others were happy to talk for much longer. Similarly, while some participants would have liked to receive more calls at the conclusion of the intervention, most participants were satisfied with the length of the programme. Some also commented on the frequency of the calls and believed that 'once a week was a good amount of calls' (P17). In terms of the modality of the intervention, while a couple of participants 'would have liked face-to-face' (P36) support, most 'liked the convenience of telephone support' (P1). One participant stated that 'telephone calls are a good way to receive social support without having to go out' (P2). Another participant liked receiving telephone support because 'even though they knew the voice, the anonymity was good' (P19).

\section{Supportive relationships developed between participants and} volunteer peers

Most participants reported that their volunteer peers were supportive and understanding. One participant stated that they 'felt they could confide in their volunteer' (P21), while another mentioned that they 'could talk about things that they couldn't talk about with other people' (P35). Participants reported finding common interests with their volunteer peer in order to build rapport and topics discussed included sport, poetry, films, music, cooking and politics. Furthermore, some participants reported 'becoming quite good friends' (P4) with their volunteer and 'looking forward to the calls' (P15), demonstrating that it was feasible for participants to develop a supportive relationship with a volunteer in this timeframe.

\section{HOW $R U$ ? is addressing a need}

A number of participants commented on the potential for HOWR $U$ ? to fill a need for 'people who are really isolated' (P23). One participant suggested that 'after discharge is when something like this is really helpful, especially if you're on your own' (P5). Another participant mentioned that 'it is empowering to have someone to talk to when you are down and know that you are not alone' (P26). Overall, participants acknowledged that taking an interest in people who may be socially isolated, lonely or showing symptoms of depression can really make a difference.

\section{Secondary outcomes}

At the end of the 3-month study, it was observed that:

- $53 \%$ of participants experienced a reduction in the level of loneliness: pre-mean and post-mean UCLA 3-item scores of 5.76 (SD 1.84) and 4.59 (SD 1.62), respectively ( $\mathrm{t}=3.32, \mathrm{p}=0.002)$.

- $68 \%$ of participants experienced fewer depressive symptoms: pre-mean and post-mean GDS 5-item scores 2.15 (SD 1.21) and 1.03 (SD 1.22), respectively $(\mathrm{t}=4.77, \mathrm{p}=0.000)$.

- While $59 \%$ of participants experienced an increase in health-related quality of life, the difference between mean EQ-VAS scores preintervention and postintervention was not significant: pre-mean and post-mean EQ-VAS scores 57.85 (SD 26.02) and 65.44 (SD 20.13), respectively $(\mathrm{t}=-1.58, \mathrm{p}=0.124)$.

\section{DISCUSSION}

This is the first study of a hospital volunteer-delivered telephone service designed to support discharged older emergency patients with symptoms of social isolation, loneliness and/or depression. This study indicated that $H O W R U$ ? was feasible and acceptable to patients and volunteers. Our results also suggested that a hospital volunteer-delivered telephone service might reduce levels of loneliness and symptoms of depression in this patient group. A limitation was that this was a relatively small cohort study in two metropolitan hospital EDs, and it was not powered for these secondary outcomes. Hence, further research with a comparative controlled trial is required to confirm the observed effects.

The overall $30 \%$ recruitment rate was reassuring, given the challenges associated with acute illness or injury and the fast-paced nature of the ED environment, ${ }^{22}$ as well as the recognised stigma with seeking or receiving support in older populations. ${ }^{23}$ Recruitment sessions were limited to 4.5-hour time periods, due to resource constraints for this feasibility study. The target of 25 patients was met at the Alfred; however, recruitment was terminated early at Cabrini due to the majority of older patients being admitted for time periods greater than 72 hours.

The rate of patient retention in HOW R U? was promising, possibly in part due to the targeted cohort's characteristics, the supportive non-intrusive nature of the intervention which enabled relative anonymity and increased privacy over the phone, ${ }^{24}$ and commencement within 72 hours of discharge.

The positive feedback was encouraging and is in common with that reported by the UK Call in Time telephone 'befriending' service for older people. Evaluation 
of this service indicated a major impact on quality of life, with participants reporting that they felt a sense of belonging, that life was worth living and they valued knowing that 'there's a friend out there ${ }^{25}$ This resonates with comments received from $H O W R U$ ? participants.

Social isolation, loneliness and depressed mood are prevalent among older people living in the community, with $12 \%$ feeling socially isolated, ${ }^{26} 50 \%$ reporting loneliness, ${ }^{27} 28$ and depressive feelings in up to $20 \%{ }^{29}$ Self-reported rates probably under-represent true levels because of an associated stigma among older people. ${ }^{29}$ Therefore, older patients with loneliness or depressive feelings are highly likely not to be identified, ${ }^{30}$ reducing the opportunity for appropriate support to be implemented in the community.

Older people presenting to ED are at an increased risk of feeling socially isolated, lonely or depressed, ${ }^{31}$ which are associated with increased re-attendance ${ }^{32}$ and negative health outcomes such as early mortality, suicide, dementia and stroke. ${ }^{33}$ These consequences have far-reaching public health impacts in terms of reduced quality of life and increased hospital use. Furthermore, with population ageing, it is likely that the number of older people at risk of social isolation and loneliness will continue to grow, as will their rates of ED use. The ED visit provides an opportunity to systematically identify social isolation, loneliness or depressive symptoms. If proven effective, implementation of peer support through $\mathrm{HOW}$ $R U$ ? should help combat the associated deleterious consequences, thereby diminishing this important public health and individual burden.

$H O W R U$ ? has the potential to reduce symptoms of depression, loneliness and social isolation among vulnerable older people, as well as to improve quality of life. Volunteers represent a significant adjunct resource for meeting some of the health and social care service needs of our more vulnerable older population. Additional benefits include the positive effects that the act of meaningful volunteering has on the peer supporter, including a positive correlation between volunteering and perceived health, and a negative correlation with depression in older volunteers. ${ }^{34}$ Maintenance of an effective high-quality volunteer service requires professional staff to coordinate and manage recruitment, training and the provision of day-to-day supervision, support and oversight; however, the use of volunteers in hospitals has been shown to be cost-effective alongside increased levels of patient satisfaction. ${ }^{35}$ Our qualitative and quantitative findings will now inform the design of a future randomised controlled trial and programme evaluation.

\footnotetext{
Author affiliations

${ }^{1}$ School of Public Health and Preventive Medicine, Monash University, Melbourne, Victoria, Australia

${ }^{2}$ Bolton Clarke Research Institute, Melbourne, Victoria, Australia

${ }^{3}$ Volunteer Services, Alfred Health, Melbourne, Victoria, Australia

${ }^{4}$ Volunteer Services, Cabrini Health, Malvern, Victoria, Australia

${ }^{5}$ Emergency and Trauma Centre, Alfred Health, Melbourne, Victoria, Australia

${ }^{6}$ Epworth Hospital, Richmond, Victoria, Australia
}

${ }^{7}$ Shenzhen International Institute for Primary Health Care Research, Shenzhen, China

${ }^{8}$ Australian National University, Canberra, Australia

${ }^{9}$ Monash University, Melbourne, Victoria, Australia

${ }^{10}$ Nursing Services and Cabrini Institute, Cabrini Health, Malvern, Australia

${ }^{11}$ Alfred Health, Melbourne, Victoria, Australia

${ }^{12}$ Warwick Medical School, University of Warwick, Coventry, UK

Contributors JAL conceived, developed the study protocol and obtained funding for the study. JD and CJB provided expertise to help design of the intervention. JAL, $A L, A C, G W, C R, D V S, D O, L B, C S, P C, J D$ and CJB contributed to refinement of the study protocol. JAL, AL, AC, GW, CR, DVS, DO, LB, CS, PC, JD and CJB contributed to the acquisition, analysis or interpretation of data. JAL drafted the manuscript. JAL, AL, AC, GW, CR, DVS, DO, LB, CS, PC, JD and CJB helped to review and revise it critically for intellectual content and approved the final version to be published.

Funding This work was supported by a grant from the Monash Partners Academic Health Science Centre.

Competing interests None declared.

Patient consent Obtained.

Ethics approval Alfred Health (432/15), Cabrini Health (12-09-11-15) and Monash University (CF15/4468-2015001934).

Provenance and peer review Not commissioned; externally peer reviewed.

Data sharing statement There are no additional unpublished data for this study.

Open access This is an open access article distributed in accordance with the Creative Commons Attribution Non Commercial (CC BY-NC 4.0) license, which permits others to distribute, remix, adapt, build upon this work non-commercially, and license their derivative works on different terms, provided the original work is properly cited and the use is non-commercial. See: http://creativecommons.org/ licenses/by-nc/4.0/

(c) Article author(s) (or their employer(s) unless otherwise stated in the text of the article) 2018. All rights reserved. No commercial use is permitted unless otherwise expressly granted.

\section{REFERENCES}

1. Aminzadeh F, Dalziel WB. Older adults in the emergency department: a systematic review of patterns of use, adverse outcomes, and effectiveness of interventions. Ann Emerg Med 2002;39:238-47.

2. Molloy GJ, McGee HM, O'Neill D, et al. Loneliness and emergency and planned hospitalizations in a community sample of older adults. J Am Geriatr Soc 2010;58:1538-41.

3. Coe RM, Wolinsky FD, Miller DK, et al. Elderly persons without family support networks and use of health services. Res Aging 1985;7:617-22.

4. Hawton A, Green C, Dickens AP, et al. The impact of social isolation on the health status and health-related quality of life of older people. Qual Life Res 2011;20:57-67.

5. Dickens AP, Richards SH, Greaves CJ, et al. Interventions targeting social isolation in older people: a systematic review. BMC Public Health 2011;11:647.

6. Choi NG, Marti CN, Bruce ML, et al. Relationship between depressive symptom severity and emergency department use among low-income, depressed homebound older adults aged 50 years and older. BMC Psychiatry 2012;12:233.

7. Mistry R, Rosansky J, McGuire J, et al. Social isolation predicts re-hospitalization in a group of older American veterans enrolled in the UPBEAT program. Unified psychogeriatric biopsychosocial evaluation and treatment. Int J Geriatr Psychiatry 2001;16:950-9.

8. Lowthian J, Straney LD, Brand CA, et al. Unplanned early return to the emergency department by older patients: the safe elderly emergency department discharge (SEED) project. Age Ageing 2016;45:255-61.

9. Lowthian JA, Straney LD, Brand CA, et al. Predicting functional decline in older emergency patients-the Safe Elderly Emergency Discharge (SEED) project. Age Ageing 2017;46:219-25.

10. Dale J, Caramlau I, Sturt J, et al. Telephone peer-delivered intervention for diabetes motivation and support: the telecare exploratory RCT. Patient Educ Couns 2009;75:91-8.

11. Paul G, Smith SM, Whitford D, et al. Development of a complex intervention to test the effectiveness of peer support in type 2 diabetes. BMC Health Serv Res 2007;7:136. 
12. Dale J, Caramlau IO, Lindenmeyer A, et al. Peer support telephone calls for improving health (Review). Cochrane Database Syst Rev 2009.

13. Dale J, Caramlau IO, Lindenmeyer A, et al. Peer support telephone calls for improving health. Cochrane Database Syst Rev 2008;4:CD006903.

14. Lowthian JA, Lennox A, Curtis A, et al. HOspitals and patients WoRking in Unity (HOW R U?): protocol for a prospective feasibility study of telephone peer support to improve older patients' quality of life after emergency department discharge. BMJ Open 2016;6:e013179.

15. Steptoe A, Shankar A, Demakakos $P$, et al. Social isolation, loneliness, and all-cause mortality in older men and women. Proc Natl Acad Sci U S A 2013;110:5797-801.

16. Hughes ME, Waite LJ, Hawkley LC, et al. A short scale for measuring loneliness in large surveys: results from two population-based studies. Res Aging 2004;26:655-72.

17. Rinaldi $P$, Mecocci $P$, Benedetti $C$, et al. Validation of the five-item geriatric depression scale in elderly subjects in three different settings. J Am Geriatr Soc 2003;51:694-8.

18. Folstein MF, Folstein SE, McHugh PR. "Mini-mental state". A practical method for grading the cognitive state of patients for the clinician. J Psychiatr Res 1975;12:189-98.

19. EuroQol Group. EuroQol--a new facility for the measurement of health-related quality of life. Health Policy 1990;16:199-208.

20. Dennis CL. The process of developing and implementing a telephone-based peer support program for postpartum depression: evidence from two randomized controlled trials. Trials 2014;15:131.

21. Ritchie J, Spencer L. Bryman AQualitative data analysis for applied policy research. In: , B R, eds. Analyzing qualitative data. London: Routledge, 1994:173-94.

22. Kendrick D, Lyons R, Christie N, et al. Recruiting participants for injury studies in emergency departments. Inj Prev 2007;13:75-7.

23. National Ageing Research Institute. Final Report. In. beyondblue depression in older age: a scoping study: NARI, 2009.
24. Heisler M, Halasyamani L, Resnicow K, et al. "I am not alone": the feasibility and acceptability of interactive voice response-facilitated telephone peer support among older adults with heart failure. Congest Heart Fail 2007;13:149-57.

25. Cattan M, Kime N, Bagnall AM. Low-level support for socially isolated older people - An evaluation of telephone befriending. In: Call in Time. Age UK. Ed. London: Help the Aged, 2009.

26. Windle K, Francis J, Coomber C. Preventing loneliness and social isolation: interventions and outcomes. London: Social Care Institute for Excellence, 2011.

27. Office for National Statistics. Measuring national well-being - older people and loneliness. London: Office for National Statistics, 2013.

28. Franklin A, Tranter B, AustraliaL. Lonelinessin Australia. In: Housing and community research unit : University of Tasmania, 2008.

29. National Ageing Research Institute. beyondblue depression in older age: a scoping study: NARI, 2009.

30. Unützer J, Katon W, Callahan CM, et al. Depression treatment in a sample of 1801 depressed older adults in primary care. J Am Geriatr Soc 2003:51:505-14.

31. Lowthian JA, Curtis AJ, Cameron PA, et al. Systematic review of trends in emergency department attendances: an Australian perspective. Emerg Med J 2011;28:373-7.

32. Rottenberg Y, Jacobs JM, Stessman J. Depression and health service utilization from age 70 to 85 : the Jerusalem Longitudinal Study. J Am Med Dir Assoc 2013;14:711.

33. Holt-Lunstad J, Smith TB, Layton JB. Social relationships and mortality risk: a meta-analytic review. PLoS Med 2010;7:e1000316.

34. Wheeler JA, Gorey KM, Greenblatt B. The beneficial effects of volunteering for older volunteers and the people they serve: a metaanalysis. Int J Aging Hum Dev 1998;47:69-79.

35. Hotchkiss RB, Fottler MD, Unruh L. Valuing volunteers: the impact of volunteerism on hospital performance. Health Care Manage Rev 2009;34:119-28. 